high-dose-rate brachytherapy. They also assessed the impact of a 6-month course of neoadjuvant or concurrent androgendeprivation therapy (ADT) in 406 of the patients. The radiation dose administered was approximately 123 Gy - the highest recorded dose ever delivered to the prostate, say the authors.

All patients had at least one poor prognostic factor (clinical stage $\geq T 2 b$, initial PSA $\geq 10 \mathrm{ng} / \mathrm{ml}$, or Gleason score $\geq 7$ ).

No differences were seen in overall survival, cause-specific survival, or biochemical control between the two groups after 5 or 8 years of follow up. Cox regression analysis, performed on unfavorable patients only, showed that ADT was associated with an increase in distant metastasis and a significant increase in prostate cancer deaths.

The authors conclude that an accelerated, hypofractionated, radiotherapy regime incorporating EBRT and high-dose brachytherapy is both effective and feasible, resulting in high survival rates.

Their findings regarding neoadjuvant ADT led the authors to suggest that delaying radiotherapy to administer ADT is "unnecessary, costly and perhaps harmful".

Tamsin Osborne

Original article Martinez AA et al. (2005) Lack of benefit from a short course of androgen deprivation for unfavorable prostate cancer patients treated with an accelerated hypofractionated regime. Int J Radiat Oncol Biol Phys 62: $1322-1331$

\section{Minimally invasive treatment of renal cell carcinoma}

Gervais and colleagues have recently reported on their experience of radiofrequency ablation as an alternative to surgery in patients with renal cell carcinoma. The findings suggest that this minimally invasive technique is useful in those for whom conventional nephrectomy is inappropriate, and that small, noncentral tumors are most likely to respond favorably to treatment.

During a 6-year period, the team performed radiofrequency ablation on 100 renal tumors in 85 patients. The mean size of the tumors was $3.2 \mathrm{~cm}$ (range $1.1-8.9 \mathrm{~cm}$ ). Multiple, overlapping ablations were carried out where needed, and repeat sessions were used if residual tumor was detected by CT or MRI after treatment.
The procedure was carried out on an outpatient basis in most cases.

Complete tumor necrosis was observed in $90 \%$ of the tumors, and the mean diameter of these successfully treated tumors was $2.9 \mathrm{~cm}$ (range $1.1-5.5 \mathrm{~cm}$ ). Multivariate analysis showed that small tumor size was an independent predictor of complete necrosis after a single ablation session, and a trend was shown between small tumor size and successful treatment after multiple sessions. Noncentral location was independently associated with complete necrosis after a single session of ablation or after multiple sessions. All exophytic tumors were completely ablated, whereas those in a central location or with central and exophytic components were successfully treated in $78 \%$ and $61 \%$ of cases, respectively.

Ruth Kirby

Original article Gervais DA et al. (2005) Radiofrequency ablation of renal cell carcinoma: part 1, indications, results, and role in patient management over a 6-year period and ablation of 100 tumors. AJR 185: 64-71

\section{Reproductive health in middle- aged and older men}

Reproductive health disorders in middle-aged and older men were the focus of a recent telephone survey carried out in Australia. The Men in Australia Telephone Survey (MATeS) also looked at related concerns, attitudes, and knowledge regarding male reproductive health.

Holden et al gathered information during a 20 min computer-assisted telephone interview from 5,990 men aged 40 years or over who had been randomly selected from all states of Australia, with stratification by age and state.

Numerous findings emerged from the study: $34 \%$ of men reported having one or more reproductive health disorder, all such disorders being most common in men aged 70 years or over. The proportion of men reporting sexual activity in the previous 12 months was lower in the older age groups, although this was still approximately $37 \%$ of men aged $\geq 70$ years. Almost $50 \%$ of participants reported having been tested for prostate problems, whereas only around $30 \%$ with significant erectile dysfunction had sought help from a health professional-the fewest men sought help in the oldest age group, and 